\title{
Linx
}

Revue des linguistes de l'université Paris X Nanterre

9 | 1997

Émile Benveniste. Vingt ans après

\section{Dialogues méthodologiques dans les Problèmes de linguistique générale}

\section{Mariagrazia Margarito}

\section{(2) OpenEdition}

\section{Journals}

Édition électronique

URL : http://journals.openedition.org/linx/1088

DOI : 10.4000/linx.1088

ISSN : 2118-9692

Éditeur

Presses universitaires de Paris Nanterre

\section{Édition imprimée}

Date de publication : 1 avril 1997

Pagination : 375-384

ISSN : 0246-8743

\section{Référence électronique}

Mariagrazia Margarito, «Dialogues méthodologiques dans les Problèmes de linguistique générale », Linx [En ligne], 9 | 1997, mis en ligne le 10 juillet 2012, consulté le 01 mai 2019. URL : http:// journals.openedition.org/linx/1088; DOI : 10.4000/linx.1088 


\title{
Dialogues méthodologiques dans les Problèmes de linguistique générale
}

\author{
Mariagrazia Margarito
}

C es notes concernent un procédé qui nous intéresse depuis quelques temps, ${ }^{1}$ à plus forte raison dans les discours scientifiques, à savoir la méthode qui se révèle et qui s'énonce, celle qui est mise en action pour la scientificité du texte lui-même. Une sorte de discours sur ou sous le discours, ou en filigrane, qui n'hésite pas toutefois à se réserver des plages d'émergence à la surface du texte. Et chaque fois qu'il y a cette générosité déictique, une sorte d'ostension ("voici la méthode que je vais suivre", "voici la méthode, et je suis cette méthode") il y a, nous semble-t-il, un souci didactique évident.

C'est donc cette didactique de la méthode qui nous occupera ici et pour laquelle le dialogue nous a paru être privilégié, même s'il n'est pas formellement actualisé, et surtout, oserions-nous affirmer, au-delà des questions-réponses que l'on trouve dans certains textes des Problèmes de linguistique générale ${ }^{2}$.

Dialogue au sens le plus global du terme, celui qui, selon les postulats de C. Kerbrat-Orecchioni, participe de l'essence du langage ("le langage verbal est donc par essence fait pour être adressé") ${ }^{3}$ puisque "Tout énoncé, même monologal est (...) virtuellement dialogal"4. Dialogue qui fait l'objet de maintes

\footnotetext{
${ }^{1}$ Cf. notre Discours en sous-main (dans "Figures" et "Palimpsestes" de G. Genette), AA.VV., Parcours et rencontres. Mélanges de langue, d'histoire et de littérature française offerts à Enea Balmas, Paris, Klincksieck, 1993, 2 vol., t. II, p. 1507-1524, et Stéréotypes, fixités ou l'éloge de la métamorphose, Actes du XXe Colloque de la Società Universitaria per gli Studi di lingua e letteratura francese, Ferrara, Centro stampa Università, 1995, pp. 169-178.

2 Dorénavant PLG. Nous citons ici l'édition de la collection Tel, Paris, Gallimard, 1993, 2 vol., t. I, 1966, t. II, 1974. Les références indiquent en chiffres romains le volume et en chiffres arabes la page.

${ }^{3}$ C. Kerbrat-Orecchioni, Les interactions verbales I, Paris, Armand Colin, 1990, p. 14.

4 Ibid. Un peu plus loin l'auteur cite Todorov citant à son tour Volochinov : "Le dialogue - l'échange des mots - est la forme la plus naturelle du langage", p. 15.
} 


\section{Mariagrazia Margarito}

références tout au long des PLG, s'inscrivant dans les apports les plus connus de Benveniste aux disciplines linguistiques: la polarité des personnes je/tu, donnée fondamentale qui lui fait souligner combien est "Unique (...) la condition de l'homme dans le langage" (I, 260), ou bien dialogue à valeur synonymique de la "condition du langage humain" $(\mathrm{I}, 60)$ - les échanges dialogiques sont inconnus des abeilles, par exemple -, dialogue comme relation première entre un sujet et son analyste (en psychanalyse) (I, 77), et encore dialogue en tant que structure nécessairement posée par l'énonciation (II, 85-8). Nous retiendrons l'affirmation à caractère général que

l'énonciation est l'accentuation de la relation discursive au partenaire, que celui-ci soit réel ou imaginé, individuel ou collectif (II, 85).

Et dialogue donc pour nous, ici, au sens d'une quasi-maïeutique.

$\mathrm{Au}$ fil des décennies, dans les commentaires sur l'oeuvre de Benveniste une des affirmations que l'on rencontre le plus souvent porte sur la rigueur, la clarté, les deux différemment conjuguées (la "rigoureuse clarté") ${ }^{5}$ de ses procédés méthodologiques.

Déjà dans son Avant-propos aux Etrennes de linguistique offertes par quelques amis à Emile Benveniste ${ }^{6} \mathrm{~A}$. Meillet annonçait, en l'étendant à un groupe de jeunes élèves, une des caractéristiques de son jeune successeur à l'Ecoles des Hautes-Etudes :

apporter du neuf, ce n'est pas appliquer à des faits connus quelque idée générale, ayant une apparence d'originalité; c'est interpréter, d'une manière exacte et personnelle, des faits recueillis de première main. ${ }^{7}$

On ne peut passer sous silence les pages consacrées à Benveniste par Roland Barthes, qui se déclarait amateur en linguistique et fier de l'être. On retrouve là, à l'avant-scène d'autres remarques sur les contenus, voire sur le style, le constat que Benveniste "n'est pas seulement un savant du langage, de la fonction langage, c'est un savant des langues"8, qu'il y a chez ce linguiste, "homme de science (..) rigoureux"9 "un savoir impeccable"10, en plus de l'admiration

\footnotetext{
5 Article de Roger-Pol Droit paru dans Le Monde à l'occasion de la mort de Benveniste, L'héritier de Saussure, 9 octobre 1976, p. 1 et 30 .

6 Paris, Librairie Orientaliste Paul Geuthner, 1928.

7 Ibid.p. VI. C'est nous qui soulignons.

8 R. Barthes, Le Grain de la Voix. Entretiens 1962-1980, Paris, Seuil, 1981, p. 202.

9 R. Barthes, Le bruissement de la langue. Essais critiques IV, Paris, Seuil, 1984, p. 194. C'est nous qui soulignons.

10 Ibid., p. 192. C'est nous qui soulignons.
} 
pour un texte, par exemple l'essai sur le système des prépositions en latin, jugé "fascinant de clarté"11.

Dans l'éloge prononcé un an après la mort de Benveniste à l'Accademia Nazionale dei Lincei Tristano Bolelli glissait, comme allant déjà de soi, et au sujet des rapports langue/société : "Emile Benveniste affronta direttamente il problema nel consueto modo rigoroso"12.

L'intérêt que les jeunes générations portent encore aux textes les plus célèbres des PLG, la découverte de pages que les étudiants avouent toujours avoir "plaisir à lire" nous ont poussée à cerner de plus près cette didactique de la méthode dont les principes et les étapes nous sont souvent annoncés par l'auteur lui-même. Là aussi réside, à notre avis et dans le cadre de la présente étude, l'originalité de Benveniste : indiquer le chemin devant soi et le chemin suivi, et non seulement les résultats obtenus.

A côté d'affirmations de démarrage, d'ouverture, d'autres pour montrer l'induction ou la déduction se faisant à petits pas :

Rien ne peut donc nous dispenser d'un examen de la distribution respective des deux pronoms je et moi $(I I, 199)$,

les pauses nécessaires dans le processus ("Cette relation au temps mérite qu'on s'y arrête, qu'on en médite la nécessité, et qu'on s'interroge sur ce qui la fonde" (II, 83), jusqu'aux paliers logiques où on tire des conclusions, on énonce des principes et on reprend l'envol pour continuer l'analyse :

Enonçons donc ce principe : tout ce qui relève du sémiotique a pour critère nécessaire et suffisant qu'on puisse l'identifier au sein et dans l'usage de la langue (...) De là résultent trois conséquences de principe (II, 222-3).

Tout cela, sans laisser de côté les suggestions pour les recherches à faire, à venir :

Bien d'autres développements seraient à étudier dans le contexte de l'énonciation. Il y aurait à considérer les changements lexicaux que l'énonciation détermine, la phraséologie (...) Il faudrait aussi distinguer l'énonciation parlée de l'énonciation écrite (II, 88).

Le problème qui se pose - et nous allons rappeler des évidences - est celui contre lequel bute tout travail se voulant scientifique, à savoir la mise en place de critères de scientificité.

\footnotetext{
11 Ibid. C'est nous qui soulignons. Dans le même article nous lisons : "il est capital (...) qu'un linguiste aussi rigoureux que Benveniste soit lui-même conscient des pouvoirs de sa discipline".

12 T. Bolelli, Emile Benveniste. Discorso commemorativo pronunciato nella seduta ordinaria del 18 novembre 1977, Roma, Accademia Nazionale dei Lincei, 1979, p. 13. C'est nous qui soulignons.
} 


\section{Mariagrazia Margarito}

Dans ses Prolégomènes à une théorie du langage, dont la traduction parut en France après la publication du premier tome des PLG ${ }^{13}$, Louis Hjelmslev spécifie, à propos de la description, qu'elle doit être non contradictoire, exhaustive et aussi simple que possible. L'exigence de non-contradiction l'emporte sur celle de description exhaustive, et l'exigence de description l'emporte sur celle de simplicité ${ }^{14}$.

Les mêmes critères, enrichis de corollaires, de mises en garde, de suggestions pour creuser toujours dans d'autres directions sont présents dans les deux volumes que nous analysons. Le dispositif didactique mis en place étale une panoplie d'éléments et de stratégies, souvent non homogènes, que nous avons essayé de relever et de regrouper.

Nous avons remarqué qu'autour d'un noyau plus proprement méthodologique, comprenant des principes (théorisation) et des éléments de méthode (des techniques) auxquels bien des études se sont intéressées ${ }^{15}$, gravite tout un ensemble hétérogène de procédés argumentatifs et stylistiques qu'il n'est pas toujours facile de cerner et qui fait, par ailleurs, la fascination de l'écriture des PLG.

Dans cet ensemble nous énumérons des assertions à valeur de vérité générale, sorte de maximes (la "frappe gnomique" selon Genette) ${ }^{16}$ concernant soit des valeurs universelles, existentielles :

il y a chez tout créateur une certaine exigence, cachée, permanente, qui le soutient et le dévore [à propos de Saussure] $(I, 33)$

La distance à parcourir a moins d'importance que la direction où s'orienter $(I, 118)$,

l'erreur acquiert autorité par la répétition (II, 164)

soit des vérités générales dans le cadre de la recherche, quelle qu'elle soit :

Bien des découvertes ont commencé par une observation (...), un désaccord dans un système, une perturbation dans un champ $(I, 35)$

Il est plus fructueux de concevoir l'esprit comme virtualité que comme cadre, comme dynamisme que comme structure. C'est un fait que, soumise aux exigences des méthodes scientifiques, la pensée adopte partout les mêmes démarches en quelque langue qu'elle choisisse de décrire l'expérience (I, 73).

\footnotetext{
13 L. Hjelmslev, Prolégomènes à une théorie du langage, Paris, éd. de Minuit, 1968-71.

14 Ibid., p. 19.

15 Cf. "Lectures d'Emile Benveniste", LINX, n² 26, 1992, sous la direction d'A. Montaut et de C. Normand.

${ }^{16}$ G. Genette, Palimpsestes. La littérature au second degré, Paris, Seuil, 1982.
} 
Ou encore, des énoncés sur le langage et les langues, et leur généralisation dans l'assertivité tient souvent lieu de définition :

C'est dans le discours, actualisé en phrases, que la langue se forme et se configure $(I, 131)$

Dans le verbe comme dans le pronom personnel, le pluriel est facteur d'illimitation, non de multiplication (I, 235)

Une langue sans expression de la personne ne se conçoit pas (I, 261).

bien avant de communiquer, le langage sert à vivre (II, 217).

Sur le plan stylistique nous notons le recours (et le terme a ici l'acception plutôt de présence que de demande d'aide) à des figures de rhétorique. La métaphore, aux nombreuses occurrences, dont un des pôles (l'autre étant un terme spécifique des disciplines linguistiques) peut être une donnée chère à la tradition classique, comme la Méduse ${ }^{17}$, ou indirectement des éléments de la nature : l'eau, le vent ("Parfois certains noms flottent entre l'adjectif et le nom d'agent", II, 114), surtout s'il est question d'emploi métaphorique de mots appartenant au lexique général.

La plupart des fois la métaphore n'a point de valeur ornementale à l'intérieur d'un discours scientifique qui se déploie et se noue, mais véritablement de moyen pour faire avancer démonstration et argumentation :

Cette condition préalable est d'importance primordiale [verbe habeo ayant en construction prédicative les deux sens "tenir" et "avoir"] : elle commande le carrefour du choix (II, 128)

[à propos de microbe] Sédillot a simplement habillé en grec une dénomination qu'il avait conçue en français (II, 168).

Autre procédé rhétorique, la personnification, dont Patrick Dahlet nous a touché mot dans sa communication. A cet égard, et en reprenant aussi les propos de Gilbert Lazard sur la dramatisation que souvent Benveniste effectuait dans ses cours, nous soulignons la véritable mise en scène des langues et du langage dans les PLG, ordinairement dans des passages à teneur quasi-narrative :

17 "On aura beau faire : cette tête de Méduse [le sens] est toujours là, au centre de la langue, fascinant ceux qui la contemplent" (I, 126).

L'oracle de Delphes aussi est rappelé : "Et voici que se ranime dans notre mémoire la parole limpide et mystérieuse du vieil Héraclite, qui conférait au Seigneur de l'oracle de Delphes l'attribut que nous mettons au coeur le plus profond du langage : Oute légei, oute kryptei 'Il ne dit, ni ne cache', alla semainei 'mais il signifie' "(II, 229). 


\section{Mariagrazia Margarito}

L'objet ainsi dénommé ne relève pas identiquement des deux classes. A l'une il appartient par nature, à l'autre il est attribué figurément. L'oiseau-mouche est bien un oiseau, mais un oiseau qui a une certaine similitude avec une mouche. Le papier-monnaie est du papier, non de la monnaie (...) Ainsi

des signes lexicaux comme "oiseau-mouche", "papier-monnaie" conjoignent deux notions, l'une de nature, l'autre de figure (II, 148).

Nous trouvons aussi des calques débouchant sur un hypertexte ${ }^{18}$

nihil est in lingua quod non prius fuerit in oratione $(I, 131)$

et tout un choix lexical à forte prégnance poétique dont le halo évocateur surgit du dénivellement entre lexique de spécialité et lexique général. Nous voulons dire par là que l'environnement linguistique où la terminologie scientifique est rigoureusement placée s'accompagne de lexèmes généraux à l'acception souvent figurée, tels que dilater, amplifier, massif, solennelle, estomper, diffus :

"nous" n'est pas un "je" quantifié ou multiplié, c'est un "je" dilaté" (...) le "je" s'amplifie par "nous" en une personne plus massive, plus solennelle et moins définie (...) l'emploi de "nous" estompe l'affirmation trop tranchée de "je" dans une expression plus large et diffuse : c'est le "nous" d'orateur ou d'auteur (I, 235)

la personne verbale au pluriel exprime une personne amplifiée et diffuse (ibid. $)^{19}$

Le style de Benveniste a d'ailleurs fait l'objet de remarques valorisantes : Barthes encore, par exemple, ${ }^{20}$ et Calvet Watkins

j'insiste toujours et néanmoins sur la beauté étonnante de ce livre [Noms d'agent et noms d'action en indo-européen], beauté qui reste et qui dure. Le monde est un endroit trop pauvre pour qu'on se passe d'un ouvrage pareil. L'espoir a besoin d'être nourri, ${ }^{21}$ Culioli :

et de critiques restrictives. Un témoignage pour tous, l'affirmation de

\footnotetext{
18 G. Genette, Palimpsestes, cit., pp. 11-12, à propos de la définition d'hypertextualité : "toute relation unissant un texte B [hypertexte] à un texte antérieur [hypotexte] sur lequel il se greffe d'une manière qui n'est pas celle du commentaire".

${ }^{19}$ C'est nous qui soulignons.

20 R. Barthes, Le bruissement de la langue, cit., p. 193 : "Les livres de savoir, de recherche, ont aussi leur 'style'. Celui-ci [PLG, t. I] est d'une très grande classe. Il y a une beauté, une expérience de l'intellect, qui donne à l'oeuvre de certains savants une sorte de clarté inépuisable, dont sont faites aussi les grandes oeuvres littéraires. Tout est clair dans le livre de Benveniste, tout peut y être reconnu immédiatement pour vrai ; et cependant aussi tout en lui ne fait que commencer".

${ }^{21}$ Emile Benveniste aujourd'hui. Actes du Colloque international du CNRS, Paris, Société pour l'Information Grammaticale, 2 vol., 1984, t. I, p. 9
} 
chaque article [dans PLG] est comme une oeuvre d'art, régie par sa propre nécessité, lisse et enclose sur elle-même [Benveniste] coule sa réflexion dans une rhétorique soigneusement maîtrisée, dans un discours un peu lointain d'homme cultivé, où la qualité même du style gêne l'analyse, où le vernis des mots (...) cache à l'occasion, la rapidité expéditive d'une réflexion qui reste courte ou qui n'est pas conduite à son terme. ${ }^{22}$

Par un retournement rhétorique bien connu, cette prudence à visée scientifique finit par être, à son tour - à son insu peut-être - élogieuse sur le plan de la fonction poétique.

Intéressantes aussi, dans cet ensemble textuel où le souci didactique nous apparaît auto-révélateur, des incises à valeur générale, sorte d'apartés qu'on peut rencontrer dans les passages les plus insoupçonnables pour ce genre de dérive, parce que non narratifs :

quel étrange destin que celui des idées, et comme elles semblent parfois vivore de leur vie propre $(I, 44)$.

En continuant sur le plan rhétorique, la présence de procédures de questionnement est de toute évidence un des dispositifs formels les plus utilisés par Benveniste. Envisagées dans une optique didactique, les questions simulent le parcours suivi par le maître se situant à la place de l'élève : en montrant un itinéraire déjà balisé il indique comment faire pour poser les jalons d'une recherche:

s'il y a histoire, de quoi est-ce l'histoire? qu'est-ce qui demeure? Qu'est-ce qui change et qu'est-ce qui demeure? $(I, 34)$

Comment est-ce que ces modèles s'enchaînent? Quelles sont les lois qui permettent de passer d'une structure syntaxique à une autre, d'un type d'énoncé à un autre? (II, 19).

Ces questions ont, en tant qu'actes langagiers, un statut assez spécial. D'une part, si on les dénombre parmi les questions oratoires ou rhétoriques, elles seraient de "vraies fausses questions", à considérer comme des assertions ${ }^{23}$. L'insistance dans leur utilisation, leur fréquence pour marquer des étapes dans l'enchaînement logique, surtout à partir de la mise à plat de fausses évidences, nous les fait assimiler aux questions didactiques, là aussi, toutefois, non sans quelques distinguos.

La dissymétrie de l'échange (un même actant pour les questions et pour les réponses) s'impose moins lorsque la question est prise dans la véhémence

22 Ibid., p. 78.

23 AA.VV., La question, sous la direction de C. Kerbrat-Orecchioni, Lyon, PUL, 1991, Introduction, p. 15. 


\section{Mariagrazia Margarito}

d'une démonstration, la question elle-même faisant figure de point de départ pour l'argumentation et/ou la démonstration :

Quelle est finalement la fonction assignable à cette distinction entre constituant et intégrant? C'est une fonction d'importance fondamentale. Nous pensons trouver ici le principe rationnel qui gouverne, dans les unités des différents niveaux, la relation de la forme et du sens (I,125-6).

Nous distinguons les affirmations présentant des principes de celles plutôt centrées sur des éléments et des praxis méthodologiques.

Parmi les principes, celui de non contradiction, plus exactement la mise en garde pour reconnaître la contradiction là où elle se cacherait, afin de ne pas tomber soi-même dans la contradiction :

On ne peut à la fois garder le cadre historique et le justifier par une définition a-historique $(I, 110)$

Le présent serait alors le présent de l'historien, mais l'historien ne peut s'historiser sans démentir son dessein (I, 245).

Une variante est la dénonciation de non-homogénéité :

Un pareil tableau [typologie des langues d'après Finck] n'est ni complet, ni systématique, ni rigoureux. On n'y trouve aucun des types si divers et si complexes des langues amérindiennes (...) De plus, les mêmes termes servent pour des caractéristiques qui n'ont pas le même sens (I, 111).

Nous insérons dans les principes de méthode la mise en garde quasi obsessionnelle contre les prétendues vérités, l'évidence (on comprend par là combien Benveniste avait dû plaire au Barthes dénonçant sans arrêt la doxa et le soi-disant naturel) :

Une autre confusion à éviter est celle qui pourrait naître de la représentation "instinctive" que nous nous formons de certaines notions (I, 172)

La question peut surprendre, comme tout ce qui a l'air de mettre en question l'évidence, mais il est parfois utile de demander à l'évidence de se justifier (I, 258).

Cet avertissement connaît toute une gamme de modulations, comme la méfiance de l'habitude ("L'habitude nous rend facilement insensibles", I, 254), du familier, de ce qui paraît simple.

Autre principe aux nombreuses occurrences dans les PLG celui de la relativisation des données, a fortiori quand il s'agit d'organisations et d'univers 
langagiers. Une ankylose conceptuelle - ô combien naturelle, celle-ci, nous guette lorsque nous voulons analyser des langues autres que la nôtre :

La double signification qu'on attribue au latin altus, comme "haut" et "profond" est due à l'illusion qui nous fait prendre les catégories de notre propre langue pour nécessaires et universelles $(I, 81)$.

Pour ce qui est des stratégies de la méthode suggérées ou mises en place, nous pensons aux processus de la comparaison des phénomènes et de leur délimitation, de la reconduction, à partir de cas particuliers, à des classes plus générales (induction), et du processus passant par déduction du général au particulier ${ }^{24}$.

A mi-chemin entre le principe et la stratégie, mêlant plutôt l'un à l'autre la prise en compte continuelle - sans crainte de dichotomie - d'une part du paradoxal dans le langage, de l'autre du paradoxe en tant que révélateur d'une évidence à interroger, ou tout simplement en tant qu'erreur de parcours.

A partir de l'affirmation bien connue :

Mais le langage est bien ce qu'il y a de plus paradoxal au monde, et malheureux ceux qui ne le voient pas $(I, 42)$

nous trouvons l'invite à apprendre à se faire au paradoxe, à l'inattendu :

On arrive ainsi à cette constatation - surprenante à première vue, mais profondément accordée à la nature réelle du langage que le seul temps inhérent à la langue est le présent axial du discours (II, 74-5),

et à ne jamais craindre de parcourir des chemins inverses (variante du paradoxe, mais aussi terme synonymique du retournement) :

[sur le terme latin orarium] notre exposé suivra l'ordre inverse de notre recherche (II, 241).

Ces démarches a contrario présupposent le départage rigoureux des niveaux d'analyse, ou de rapports supposés bien connus

Est-ce simplement parce qu'elle est le système le plus commun, celui qui a le champ le plus large, la plus grande fréquence d'emploi et - en pratique - la plus grande efficacité? Tout à l'inverse : cette situation privilégiée de la langue dans l'ordre pragmatique est une conséquence, non une cause de sa prééminence comme système signifiant (II, 63).

\footnotetext{
24 Cf. LINX, n 26, 1992, cit., notamment les essais de A. Montaut, "La méthode de Benveniste dans ses travaux comparatistes : son discours et son sujet", pp. 109-135, et de M. Fruyt, "Les principes méthodologiques d'Emile Benveniste dans Noms d'agent et noms d'action en indo-européen", pp. 159170.
} 
Mariagrazia Margarito

Ces traits didactiques que nous avons mis en relief ici parcourent bien des pages des PLG, sans être obligatoirement présents dans tous les textes. Reste, en outre, l'extraordinaire qualité de l'écriture de Benveniste, sous-tendue par la capacité d'un émerveillement ininterrompu, et qui s'énonce lui aussi :

Ainsi se constitue (...) un répertoire vaste, toujours ouvert, de composés descriptifs, instruments de la classification et de la nomenclature, aptes à devenir dénominations scientifiques ou épithètes poétiques et qui (...) entretiennent cette activité métamorphique, peut-être le travail le plus surprenant de la langue (II, 62). 